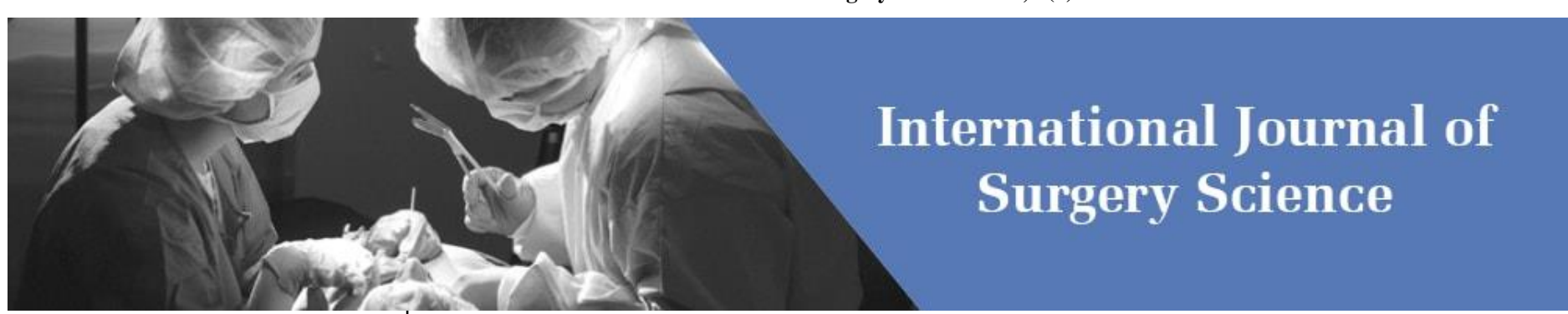

E-ISSN: 2616-3470

P-ISSN: 2616-3462

(C) Surgery Science

www.surgeryscience.com

2021; 5(4): 144-150

Received: 16-08-2021

Accepted: 18-09-2021

Dr. Akshay Kalavant B

Assistant Professor, Department of

Pediatric Surgery, Shri

Dharmasthala Manjunatheshwara

University, Dharwad, Karnataka,

India

Dr. Shreesha Nayak

Department of Emergency

Medicine, All India Institute of

Medical Sciences, New Delhi,

Delhi, India

Dr. Venkatesh Annigeri M

Department of Pediatric Surgery,

Shri Dharmasthala

Manjunatheshwara University,

Dharwad, Karnataka, India
Corresponding Author: Dr. Akshay Kalavant B

Assistant Professor, Department of

Pediatric Surgery, Shri

Dharmasthala Manjunatheshwara

University, Dharwad, Karnataka,

India

\section{A retrospective comparative study to determine the factors leading to poor outcome in operated cases of esophageal atresia and trachea-esophageal fistula: An audit}

\author{
Dr. Akshay Kalavant B, Dr. Shreesha Nayak and Dr. Venkatesh Annigeri \\ M
}

DOI: https://doi.org/10.33545/surgery.2021.v5.i4c.777

\section{Abstract}

Aim: To determine the different factors leading to poor outcome in operated case of esophageal atresia and trachea-esophageal fistula (EA-TEF).

Material and Methods: This is $a$ retrospective non-randomized comparative study. All the patient who presented with EA-TEF to us were included in the study. The patients who were not operated and patient who were discharged against the advice were excluded from the study. Patients were divided in to two groups. Group-A- Those who have survived after the surgery-39 and Group-B those who expired after the surgery-38. Various parameters were considered in relation to pre-operative, intra-operative and postoperative factors. To name few antenatal findings, maternal hemoglobin, gestational age, gap between both the end, duration of procedure, need for mechanical ventilation, early start of NG feed, need for blood products and postoperative complications.

Results: Preoperative factor which had significant negative impact on survival were antenatally diagnosed polyhydramnios, maternal anemia, gestational age and associated severe cardiac anomalies. Mean operating time was more in group-B. Whenever surgeons or anaesthesiologists decided to continue postoperative ventilation the outcome was poor- 34 needed post-operative ventilation in group-B versus 12 in group A. Need for blood transfusion and need for platelets transfusion did not show significant difference among two groups but the need for plasma transfusion had poor survival. SIRS/sepsis played important determining factor in survival of EA-TEF. Patients who could be fed early, had better survival. Pneumothorax, pneumonia and leak didn't show significant difference in survival.

Conclusion: Different factors before, during and after the surgery have impact on outcome of EA-TEF. We should be able to formulate prognostic score which is based our local needs and available resources.

Keywords: esophageal atresia, tracheoesophageal atresia, poor survival in TEF

\section{Introduction}

Management of Esophageal atresia (EA) and tracheoesophageal fistula (TEF) have come a long way which, was once considered as a disease incompatible with life in previous century, now claims near $100 \%$ survival in good weight babies without any cardiac defects in the last eight decades ${ }^{[1]}$. The EA-TEF incidence is about 1 in $2500-4000$ live births ${ }^{[2]}$. Although the mortality has reduced drastically, morbidity and complications remain high. Management of complications require lot of resources. India documents about 18000 cases every year ${ }^{[3]}$. Only about $10 \%$ of the patients are able to reach the tertiary care center. Most of the patients reach the hospital late and unable to make up in the survival list. Overall survival of the treated cases of EA-TEF is $40-55 \%$ in India ${ }^{[4-8]}$. Gestational age and birth weight are fading away from prognostic factors in the western countries. Developing countries still relies on it for prognostication. Now there are many literatures about the survivors of EA-TEF reaching the adulthood ${ }^{[9,10]}$. In last 6 decades the survival for non-syndromic children have been in rise from $36 \%$ to $95 \%$ [11, 1]. But the survival in the developing countries is still low. Maternal nutrition, perinatal care, cost of the treatment and non-availability of basic medical and surgical care are the factors affecting the survival in low- and middle-income nations.

Various prognostic criteria are laid down in the literatures, but cannot be used in developing countries and we need to develop our own criteria to address our needs and limitations ${ }^{[12.13]}$. 
Here is an attempt to outline some of the preoperative, operative and postoperative factors which can be used to determine mortality during the management of EA-TEF.

\section{Material and Methods}

This is retrospective comparative study of consecutive patient undergoing surgical repair for EA-TEF between May 2015 to June 2019. One hundred eight patients were operated between the period of 2015 and 2019. Seventy-seven patients were included in the study. Eighteen parents had discharged their child due to economic contains. Seven of them had been operated. They have been excluded from the study.

\section{Inclusion Criteria}

The patients born in our institution, who were diagnosed to have EA-TEF Babies who were referred to our institution with diagnosis of EA-TEF Patient referred to our institution who were not the diagnosed of EA-TEF but diagnosed to have the same in our institution.

\section{Exclusion criteria}

Patients of EA-TEF who have not undergone surgical repair for any reason Patients who were discharges against medical advice Patients whose data were incomplete or missing from the medical records Patients with no follow-up or non-traceable. The patients were initially resuscitated in Neonatal intensive care unit (NICU). Chest and abdominal radiograph were taken with infant feeding tube in the upper esophageal pouch to confirm and identify the type of EA-TEF. Resuscitation was performed for initial 24-72 hours and posted for operative procedure on a semi elective basis. Right postero-lateral thoracotomy was performed in types other than Type A and type $\mathrm{E}$ as identified in radiographic examination. Extrapleural approach was taken for the surgery except in case of pleural rupture where transpleural route was used. Primary esophageal anastomosis was attempted in all the case of thoracotomy. We place a chest tube in all the cases of primary anastomosis. In case of failed primary anastomosis due to long gap; fistula ligation, cervical esophagostomy and feeding gastrostomy (CEFG) was performed. We follow the protocol of extubating the patients after the procedure. Post-operative mechanical ventilation was provided for the patient in case of poor respiratory efforts, poor general health as assessed by anesthesiologists or on surgeons request, in case the anastomosis was under significant tension.

This study compares various pre-operative, intra-operative and post-operative factor that makes a difference among the patients who have survived and the ones who have not. There are two group in the study

Group A: Included the 39 patients who have survived the surgery (Survivors)

Group B: Included 38 patients who expired after the surgery (Non-survivors) following parameters were compared between the two groups

\section{Pre-operative}

1. Mode of delivery

2. Birth weight of the baby

3. Gender

4. Whether baby is born in our institution or born outside and later transported

5. Antenatally detected polyhydramnios or absence of stomach bubble

6. Presence of maternal anemia (Hemoglobin value less than $10 \mathrm{~g} / \mathrm{dL}$ in venous blood)

7. Gestational age at birth

8. Presence of respiratory distress at birth

9. Time of diagnosis of EA-TEF (antenatally or postnatally or if missed initially)

10. Associated cardiac anomalies or VECTRAL associations,

11. Number of patients requiring mechanical ventilation before the surgical procedure.

\section{Intra operative factors}

1. Duration between birth and surgery

2. Type of EA-TEF

3. Gap between proximal and distal pouch

4. Intra-operative adverse events

5. Patients needing postoperative ventilation

6. Duration of surgical procedure

\section{Post-operative factors}

1. Development of sepsis and septic shock

2. Need for blood products after the surgery

3. Starting of feed after the surgery

4. Development of pneumonia or pneumothorax

5. Documentation of any leak

6. Rate of sepsis and need for inotropic support

Statistical methods: Descriptive analysis was carried out by frequency and proportion for categorical variables. Chi-squared test was used to compare the categorical parameters. Kolmogorov-Smirnov test was used to check the normality of the data. The normally distributed continuous variables were presented in terms of mean and standard deviation and unpaired sample t-test was used to compare the two groups. The nonnormally distributed data was presented in terms of median (IQR) and Mann-Whitney U test was used to compare two groups. $\mathrm{P}$ value less than 0.05 was considered statistically significant. Data Analysis was performed using SPSS Software version 22 (IBM, Inc.).

\section{Results}

Among the 77 patients included in the study, overall survival was $50.64 \%$. The number of male neonates in the study group were $53.02 \%$ but there was higher survival noted among female babies $(63.3 \%)$. Male neonatal survival being 39.0\% (Table 1).

The mean birth weight and gestational ages were comparable in both the groups. Group B consisted of 5 early preterm babies compared to only 1 in group A. The number children referred to us from other hospitals were similar in both the groups. The mean age at referral was also similar. Number of children with respiratory distress at birth were similar in both the groups. Among them 5 patients in group A and 6 patients in group B required preoperative ventilatory support. There was a difference of 10 hours in mean time between the birth and surgery among two groups. In Group a baby was taken up for surgery after 50.79 hours whereas it was 60.42 hours in Group B. It didn't show statistical significance. Preoperative oral feeding in a child didn't show adverse outcome. Fourteen babies those who have been fed preoperatively have survived, whereas 6 patients were fed orally preoperatively in group B. Mothers of the babies in group B were anemic in $52.6 \%$ of the cases compared to $20.5 \%$ of the mothers in group A which showed statistical significance. Fifteen percent of patients in Group A had antenatally detected polyhydramnios, whereas $50 \%$ mother 
of the babies in group B had polyhydramnios. Mode of delivery didn't have an impact in the outcome. Cardiac anomalies were seen more in group A, but number of serious cardiac anomalies were noted more in group B. Following cardiac anomalies were seen in survival group atrial septal defect (ASD) in 15, patent foramen ovale (PFO) in 10, patent ductus arteriosus (PDA) in 8 and ventricular septal defect in (VSD) 4. Patient in non-survivor group showed following cardiac anomalies. ASD in 9, PFO in 4, PDA in 6, VSD in 1. There was also one patient with AV canal defect and one with hypoplastic left ventricle. Right sided aortic arch was noted in 2 patient of group B and one patient of group A. The vertebral anomalies were more common in group B (5 cases) compared to only 1 in group A. Numbers were too small to draw any conclusion. Other anomalies included anorectal malformation; 4 in group A ( 2 cloaca) and 2 in group B, skeletal deformity (commonly being poly dactyl); 5 in group A and 6 in group B which also included genu recurvatum and hypoplastic radius, one in each group. One patient had duodenal atresia but didn't survive the two surgeries.

Five parameters were noted in intra-operative predictive factors namely: type of EA-TEF, gap between the proximal and distal pouches, type of repair performed, duration of the surgical procedure, need for post-operative ventilatory support or any intraoperative adverse events. Seven patients in group A had Type A EA whereas only 1 patient had such disease in group B. These patients had undergone CE-FG. Group B also had type D EA-TEF which was initially missed during first surgery and later recognized in postoperative period by flexible bronchoscopy for recurrent respiratory distress. The type $\mathrm{C}$ was common type of EA-TEF seen in both the groups. There were 36 patients in group B, 7 requiring CE-FG for large gap found after thoracotomy. Only 4 patients among 33 in group A required CEFG. Twelve patients from group A needed postoperative mechanical ventilation compared to 34 patients in group B. Surgeons had asked for elective ventilation due to increased tension over the anastomotic site in one patient in group A compared to 9 patients in group B. The mean duration of surgery in group B was significantly higher.
Further post-operative variables were compared among the two groups. About half of the patients in each groups developed features of systemic inflammatory response syndrome. Seven patients in the group A developed the circulatory shock requiring the triple inotropic support and 31 patients required inotropic support in group B. The need for blood transfusion in postoperative period did not have any impact on the outcome of the patients. Patients in group B required more platelet transfusion although that was not statistically significant but patient requiring fresh frozen plasma for coagulopathy had worse outcome. The survival was noted to be more in the patients where the NG feeding was started within one week of the surgical correction. Early feeding was started only in less than quarter number of patients in group B. Development of postoperative pneumonia, pneumothorax and documented leak did not have any impact on the survival. None of the patients underwent repeat thoracotomy or late diversion procedures. Waterston scoring correlated significantly among group A and group B patients whereas, there was no significant difference between the Spitz's grading scheme among survival and non survivors.

Six deaths (two male and four female) were noted within one year of surgery. Two patients died after one month of discharge, both were sudden deaths and exact reasons were not known in them. Two babies died of respiratory complications after 3 months of discharge. One child died due to diarrhea related illness and malnutrition. One patient died at one year of age as a post-operative complication of gastric pull up surgery for long gap type C EA- TEF.

One patient had undergone esophageal diverticulum excision. Two patient required anti reflux surgery. Four patients had anastomotic stenosis which resolved with two dilatations and two more patients required surgery for which patient didn't turn up. One patient had poor weight gain with no surgery related complication. Total of 3 patient had undergone gastric pull up procedure in our institution for long gap type $\mathrm{C}$ deformity and one for type A EA.

Table 1: Various pre-operative, operative and post-operative parameters that were compared among group A and group B

\begin{tabular}{|c|c|c|c|c|}
\hline Sl. No. & Parameters & Group A (Survivor) & Group B (Non-survivor) & P-value \\
\hline \multicolumn{5}{|c|}{ Pre-operative parameters } \\
\hline 1 & Mode of delivery- Caesarean section & $9(23.07 \%)$ & $9(23.6 \%)$ & - \\
\hline 2 & Preterm neonates ( $<37$ weeks) & $8(20.5 \%)$ & $10(26.3 \%)$ & 0.342 \\
\hline & \multicolumn{4}{|c|}{ Gender } \\
\hline & Male child & $16(41 \%)$ & $25(65.8 \%)$ & \multirow{2}{*}{0.029} \\
\hline & Female child & $23(59 \%)$ & $13(34.2 \%)$ & \\
\hline \multirow[t]{2}{*}{4} & Neonates referred from outside & $22(56.4 \%)$ & $23(60.5 \%)$ & 0.714 \\
\hline & Mean age at referral (in hours) & 32.09 & 32.47 & $>0.05$ \\
\hline 5 & Respiratory distress at birth & $18(46.2 \%)$ & $14(36.8 \%)$ & $>0.05$ \\
\hline 6 & Maternal nutritional status (anemia) & $8(20.5 \%)$ & $20(52.6 \%)$ & 0.003 \\
\hline 7 & Antenatal polyhydramnios & $6(15.3 \%)$ & $19(50 \%)$ & $<0.010$ \\
\hline 8 & Orally fed before the surgery & $14(35.8 \%)$ & $9(23.6 \%)$ & 0.242 \\
\hline 9 & Presence of cardiac anomaly & $20(51.2 \%)$ & $15(39.4 \%)$ & 0.298 \\
\hline 10 & Preoperatively intubated & $5(12.8 \%)$ & $6(15.7 \%)$ & $>0.05$ \\
\hline 11 & Vertebral anomalies & $1(2.5 \%)$ & $5(13.1 \%)$ & - \\
\hline 12 & Duration between birth and surgery (in hours) & 50.79 & 60.42 & 0.992 \\
\hline \multicolumn{5}{|c|}{ Operative parameters } \\
\hline 1 & Duration of surgery (in minutes) & 91.03 & 112.97 & $<0.01$ \\
\hline 2 & Long gap $(3 \mathrm{~cm})$ & $4(10.2 \%)$ & $8(21.05 \%)$ & 0.038 \\
\hline 3 & Shifted to NICU intubated after the surgery & $12(30.7 \%)$ & $34(89.4 \%)$ & $<0.01$ \\
\hline 4 & & Type of TEF (Gross classification) & & \\
\hline & Type A & 7 & 1 & \\
\hline & Type C & 32 & 36 & \\
\hline & Type D & 0 & 1 & \\
\hline
\end{tabular}




\begin{tabular}{|c|c|c|c|c|}
\hline & Type B and E & 0 & 0 & \\
\hline \multicolumn{5}{|c|}{ Post-operative parameters } \\
\hline 1 & Development of SIRS & $19(48.9 \%)$ & $17(44.7 \%)$ & 0.726 \\
\hline 2 & Development of circulatory shock requiring inotropes & $7(17.9 \%)$ & $31(81.5 \%)$ & $<0.01$ \\
\hline 3 & Positive blood cultures & $19(48.7 \%)$ & $16(42.1 \%)$ & 0.560 \\
\hline 3 & Patients requiring blood transfusion & $10(25.6 \%)$ & $11(28.9 \%)$ & 0.751 \\
\hline 4 & Patients requiring FFP transfusion & $1(2.5 \%)$ & $9(23.6 \%)$ & 0.021 \\
\hline 5 & Patients requiring Platelet transfusion & $3(7.6 \%)$ & $9(23.6 \%)$ & 0.150 \\
\hline 6 & Fed the baby before 7 days of surgery & $24(61.5 \%)$ & $9(23.6 \%)$ & $<0.01$ \\
\hline 7 & Pneumonia & $4(10.2 \%)$ & $6(15.7 \%)$ & 0.470 \\
\hline 8 & Pneumothorax & $4(10.2 \%)$ & $9(23.6 \%)$ & 0.116 \\
\hline 9 & Leak & $8(20.5 \%)$ & $6(15.7 \%)$ & 0.526 \\
\hline
\end{tabular}

\section{Discussion}

Success story of surgical correction of EA-TEF have many contributors. Landmark paper from Thomas Lanman in 1940 described the death of all the 32 patients of EA-TEF, where 30 patients had undergone thoracotomy and primary esophageal anastomosis [14]. The paper taught us that the early and aggressive resuscitation followed by surgery is the only ray of hope to avoid aspiration pneumonia, dehydration and sepsis in these newborns. Due to the availability of well-trained pediatric surgeons, surgical technique is merely a cause of poor mortality in the disease. Availability of transport facility and availability of pediatric surgery care along with neonatal critical care facility are determining factors in the survival of the patient ${ }^{[15]}$. Some of the centers in India have reported delay in referral and development of hypothermia due to improper transportation as attributing factor for the high neonatal mortality in such groups ${ }^{[16]}$. In our series, there was no significance noted in survival among the patients who were born in our institution and who were referred from other hospital. Optimal time to perform the surgical procedure is within first 24 hours of birth ${ }^{[17]}$. Our mean operating time was 50 hours in survival group and was more than sixty hours in non survivors. Probably early intervention after aggressive optimization would have shown improved survival in our series. Most of the deaths takes place in immediate postoperative period. Mean duration death the surgery was 6.10 days. Most of these deaths are caused due to pneumonia, postoperative leak and sepsis. In our series, postoperative pneumonia and leak did not show statistically significant impact in mortality as early postoperative sepsis surpassed the other factors. Sharman et al. have shown that about $25 \%$ of the deaths take place in late follow-up periods caused mainly due of late deaths are respiratory complication such as hyper-reactive airways and tracheomalacia. Other etiologies include chromosomal anomalies, aspiration secondary to gastroesophageal reflux and sudden unexplained deaths ${ }^{[18]}$. We also have faced 6 deaths $(15.3 \%)$ with-in 1 year follow-up period.

Long gap is considered as independent risk factor in survival of the EA-TEF patients and have suggested it as independent prognostic criteria by Upadhyaya et al. ${ }^{[4]}$. Long gap is described as difference of more than three vertebral segments between the proximal and distal esophageal segments. The study showed that the mortality ranges from $50-80 \%$ and stricture rate ranges between $75-100 \%$ when the gap exceeds more than two centimeters. The long gap is associated with more mortality. $\mathrm{R}$ $\mathrm{K}$ Tandon et al. also have documented $53 \%$ mortality rate in patient with long gap anomaly ${ }^{[15]}$. Our study also showed significantly more mortality rate in long gap EA-TEF. In our study mortality in long gap EA-TEF was $66.6 \%$.

Antenatal detection rate of the anomaly is still low, which is 13.3- 32\% in literature. Fetal ultrasonography has sensitivity of $25-53 \%$ and positive predictive value of $35 \%$ which are comparatively low. Detection of polyhydramnios is higher in pure esophageal atresia ${ }^{[2,19,20]}$. Absence of stomach bubble was noted in 8 of our patients (five in group B). Significantly high rate of polyhydramnios was noted in $50 \%$ of our patients of nonsurvivors compared to $15 \%$ in case of survival. There is no direct evidence relating the polyhydramnios to the mortality in the literature. But prenatally diagnosed EA-TEF had higher mortality compared to one who were not detected prenatally [21] There was also correlation between the prenatally diagnosed EA-TEF and other congenital anomalies. Most of the study do not represent any gender differences, but in our study the number of male patients were more compared to the female counterpart. Such findings have also been identified in some studies ${ }^{[15,17,22-24]}$. This could be attributed to preference to male child in our country and increasing feticide leading to hospital admission bias. The survival was seen more in female child. Although no reasoning could be made, Julia et al. suggested that boys had more morbidities and more hospital stay compared to the girl child. Boys were also observed to have more polyhydramnios ${ }^{[25]}$. In our study mortality of male children was $60.9 \%$.

Commonest postoperative complication mentioned in literature is leak in immediate postoperative cases. Late postoperative complications include stenosis, gastro-esophageal reflux, tracheomalacia and dysphagia. In our series post-operative leak was very low. Only two of our patients required anti-reflux surgery which is very low compared to western literature which is as high as $23 \%-60 \%$ [1, 26, 27]. GER is more common in pure esophageal atresia. Only about half of the patients with GER need anti-reflux surgery. Thoracoscopic repair may improve the reflux rate due to better visualization of esophageal ends and preservation of vagus nerve ${ }^{[28]}$. Authors have predicted high mortality rate in patient with severe congenital cardiac problem. The predicted mortality is as high as $50 \%$ especially if is associated with low birth weight. Most often both the factors are interrelated ${ }^{[26]}$. We also found that, although the number of cardiac involvements were noted more in survival group; severe cardiac anomalies were more in non-survival groups.

The overall morbidity in TEF patients is high even in the center of excellence with complication rate up to $67 \%{ }^{[1,12]}$. But these patients are being saved due to their health resources. Raising treatment cost in case of complications and reluctance to continue the treatment has increased the rate of postoperative deaths and discharges against advices in our studies. Many of our patient had taken their babies against medical advice owing to the high treatment cost. Government health sectors are unable to handle the burden of the diseases in India. Hence, Indian union government has recently added a new insurance scheme, applicable even to the private health sectors which were not available for congenital anomalies in the past. The main goal of the insurance has been to provide basic medical care and improve the national average of neonatal mortality rate to 12 per 
1000 from existing 25/1000. It has emerged as world's largest insurance package. This might help in providing the treatment for the underprivileged population ${ }^{[29]}$. Rattan et al. in their study have pointed out the improvement in their outcome since 2006, after the implementation of government program such as National rural health mission in government health sectors ${ }^{[30]}$.

Common cause of high mortality in our study was septicemia related complications [13]. Positive blood culture was documented in 35 out of 77 patients. Klebsiella pneumonia infection is the commonest infection in neonatal intensive care unit across the world which is about $41 \%$ with mortality of $68 \%$. Our series also showed Klebsiella sepsis in $31.4 \%$ patients with $63 \%$ documented mortality ${ }^{[31]}$. Circulatory shock was noted in 31 neonates in group B and it was seen in just 5 in group A patients suggesting that need for inotropic support in septic shock is associated with poor outcome. A similar study conducted in our institution, done between 2009 to 2014 also suggested that septicemia was common cause of death in postoperative cases ${ }^{[32]}$. There are many studies which have compared their outcome among initial experience in their department with their late experiences. Most of the studies have showed significant improvement in their survival rates [22, 33, 34]. But there has been a reduction in the survival of the patient in our series. The reason could be non-availability of highly dedicated anesthesiologists, who were present in previous period. This can also be identified by high rate of intraoperative anesthesia complications. Deficiency of neonatologist and dedicated surgical nurse in our institution tend to continue as previous ${ }^{[13]}$. Patients who were not able to be extubated after the procedure had high rate of mortality. In our study nearly $90 \%$ of the patient who died were sifted to NICU on ventilator compared to survival group, where $30 \%$ subjected to postoperative ventilation. Montreal classification scheme considers ventilator dependency along with the cardiac anomalies, as one of the risk factors for mortality ${ }^{[35]}$. Maternal nutrition also plays an important role in the well-being of the mother and child ${ }^{[36]}$. Anemia is one of the important indicators of maternal nutrition and maternal care receptivity during the pregnancy ${ }^{[37]}$. Maternal anemia in low- and middle-income countries varies between $42-68 \%$ and perinatal mortality raised up to $90 / 1000$ babies born to such mothers ${ }^{[38]}$. Similar findings were noted in our series where $51.2 \%$ of the mothers were anemic in group B compared to $21.1 \%$ in group A. This factor is often ignored when we discuss about the mortality in major congenital anomalies such as EA TEF. Need for blood transfusion in neonatal period, use of umbilical catheter and unable to feed the child with human breast milk are other risk factors in development of neonatal sepsis, all the risk factors were present in our patients. Blood transfusion and platelet transfusion did not show significant difference between two groups but plasma transfusion showed significant difference in survival and non-survival group. Feeding before surgery is associated with poor outcome due to aspiration pneumonia. But our study didn't show the significant change in the both the groups. The survival chances were high when we were able to start enteral feed within a week after the surgery. This could be correlating with the general condition of the patient, as most of the patient had either died were too sick to start the nasogastric feed in group B.

Other centers in India have also shown poor survival in case of EA-TEF ${ }^{[6,7,15,16]}$. Reason for such a poor outcome have been shown as pneumonia, sepsis, long gap disease, poor transportation facility, shortage of tertiary care center for catering the disease, shortage of nursing staff for meticulous care of these neonates. One of the centers who had poor survival of TEF have shown improvement after motivation of the nursing staff and pediatric colleagues. After witnessing initial improvement in survival, the motivated team was able to provide much better results showing the importance of dedicated team work ${ }^{[39]}$. Various classification systems have been considered in predicting the survival. Most of them are retrospective factor used in estimating the mortality. It helps us in counselling the parents about the survival of the patients. It also helps in comparing the institutional outcome with other centers. Waterston criteria was the initial system used for prognostication. As the survival have increased, Spitz criteria become popular. Now clinicians are seeking for newer systems as birth weight is no more a challenge for survival. But we have found that Waterston criteria suits better in our set-up (Table 2). Our present results were the one that were achieved by the developed countries 3 to 4 decades ago. Agarwala et al. have suggested that primary care physicians, pediatricians, staff nurses need to be trained to identify the EA-TEF with the help of videos presentations and workshops as these are the people who come in contact with patients. General public also has to be sensitized about the disease and emphasis has to be laid on proper transportation of the patient to the tertiary center ${ }^{[16]}$. Our results were very poor compared to the western standards. Hence information from this study can only be used in assessing factors related to mortality in low- and medium-income countries. The patients were treated by different pediatric team, anesthesiology team could be the limitation of the study. The late postoperative complications such as GER, stricture, tracheomalacia are far less reported than other authors. This could be attributed to survival of only low risk babies.

Table 2: Comparison of Waterston and Spitz grading Schema in our patients

\begin{tabular}{|c|c|c|c|c|c|c|}
\hline & \multicolumn{3}{|c|}{ Waterston criteria(grade) } & \multirow{2}{*}{ Total } & \multirow{2}{*}{ P value } \\
\hline & & $\mathbf{A}$ & B & $\mathbf{C}$ & & \\
\hline \multirow{4}{*}{ survival } & Groun P & 9 & 13 & 16 & 38 & \multirow{6}{*}{0.007} \\
\hline & Group B & $23.7 \%$ & $34.2 \%$ & $42.1 \%$ & $100.0 \%$ & \\
\hline & \multirow{2}{*}{ Group A } & 6 & 27 & 6 & 39 & \\
\hline & & $15.4 \%$ & $69.2 \%$ & $15.4 \%$ & $100.0 \%$ & \\
\hline \multirow{2}{*}{\multicolumn{2}{|c|}{ Total }} & 15 & 40 & 22 & 77 & \\
\hline & & $19.5 \%$ & $51.9 \%$ & $28.6 \%$ & $100.0 \%$ & \\
\hline & & \multicolumn{3}{|c|}{ Spitz criteria(grade) } & \multirow{2}{*}{ Total } & \multirow{8}{*}{0.86} \\
\hline & & 1 & 2 & 3 & & \\
\hline \multirow{4}{*}{ survival } & \multirow{2}{*}{ Group B } & 25 & 11 & 2 & 38 & \\
\hline & & $65.8 \%$ & $28.9 \%$ & $5.3 \%$ & $100.0 \%$ & \\
\hline & \multirow{2}{*}{ Group A } & 26 & 12 & 1 & 39 & \\
\hline & & $66.7 \%$ & $30.8 \%$ & $2.6 \%$ & $100.0 \%$ & \\
\hline \multirow{2}{*}{\multicolumn{2}{|c|}{ Total }} & 51 & 23 & 3 & 77 & \\
\hline & & $66.2 \%$ & $29.9 \%$ & $3.9 \%$ & $100.0 \%$ & \\
\hline
\end{tabular}

\section{Conclusion}

Sepsis is main contributory factor for high mortality of EA-TEF patients in our study. We have observed that polyhydramnios, maternal anemia, patient requiring post-operative ventilatory support, septic shock requiring inotropic support, coagulopathy requiring plasma transfusion have high mortality. Whereas female gender, lesser anesthesia and procedure time, able to start the feed within a week after surgery have favorable outcome. Availability of dedicated pediatric anesthesiologist, round the clock neonatologist and surgical nurse can also make a significant impact in the survival. Government initiatives and availability of insurance schemes can reduce the economic burden for the family and improve the outcome. We should be 
using the prognostic criteria bases on our needs and limitations; and it is wise to formulate our own prognostic criteria. Good maternal nutrition can improve neonatal survival and out-come of EA-TEF.

\section{Acknowledgements}

Dr. Archana Bhat for helping out with statistics

\section{Declarations \\ Funding: None}

\section{Conflict of interest: None}

Ethical approval: Taken - SDMCDS IEC. No. 2021/Medical/ Pediatric/S/03 dated 15-06-2021

\section{References}

1. Sinha CK, Haider N, Marri RR et al. Modified prognostic criteria for oesophageal atresia and tracheo-oesophageal fistula. Eur J Pediatr Surg 2007;17:153-7.

2. Lal DR, Gadepalli SK, Downard CD et al. Midwest Pediatric Surgery Consortium. Perioperative management and outcomes of esophageal atresia and tracheoesophageal fistula. J Pediatr Surg 2017;52:1245-51.

3. Upadhayaya P. Esophageal atresia in India. In: Willital GH, Nihoul-Fekete C, Myers NA, editors. Management of esophageal atresia: Diagnosis, therapy, complications and late results. Munich: Urban and Schwarzenberg 1990, 2832.

4. Upadhyaya VD, Gangopadhyaya AN, Gupta DK et al. Prognosis of congenital tracheoesophageal fistula with esophageal atresia on the basis of gap length. Pediatr Surg Int 2007;23:767-71.

5. Davari HA, Hosseinpour M, Nasiri GM et al. Mortality in esophageal atresia: Assessment of probable risk factors (10 years' experience). J Res Med Sci 2012;17:540-2.

6. Kumar P, Ojha P. Oesophageal atresia: presentation and. outcome in Bihar. Indian Journal of Surgery 1999;6:29-32.

7. Bhatnagar V, Agarwala S, Bajpai M et al. Results of treatment of esophgeal atresia. J Indian Assoc Pediatr Surg 1997;2:55-8.

8. Sharma AK, Shukla AK, Prabhakar G et al. Oesophageal atresia: tragedies and triumphs over two decades in a developing country. Int Surg 1993;78:311-314.

9. Friedmacher F, Kroneis B, Huber-Zeyringer A et al. Postoperative Complications and Functional Outcome after Esophageal Atresia Repair: Results from Longitudinal Single-Center Follow-Up. J Gastrointest Surg 2017;21:92735 .

10. Acher CW, Ostlie DJ, Leys CM et al. Long-Term Outcomes of Patients with Tracheoesophageal Fistula/Esophageal Atresia: Survey Results from Tracheoesophageal Fistula/Esophageal Atresia Online Communities. Eur J Pediatr Surg 2016;26:476-80.

11. Manning PB, Morgan RA, Coran AG et al. Fifty years' experience with esophageal atresia and tracheoesophageal fistula. Beginning with Cameron Haight's first operation in 1935. Ann Surg 1986;204:446-53.

12. Seo J, Kim DY, Kim AR et al. An 18-year experience of tracheoesophageal fistula and esophageal atresia. Korean $\mathbf{J}$ Pediatr 2010;53:705-10.

13. Li XW, Jiang YJ, Wang XQ et al. A scoring system to predict mortality in infants with esophageal atresia: A casecontrol study. Medicine (Baltimore) 2017;96:e7755.
14. Lanman TH. Congenital atresia of the esophagus: a study of thirty-two cases. Arch Surg 1940;41:1060-83.

15. Tandon RK, Sharma S, Sinha SK et al. Esophageal atresia: Factors influencing survival - Experience at an Indian tertiary centre. J Indian Assoc Pediatr Surg 2008;13:2-6.

16. Agarwala S, Bhatnagar V, Bajpai $\mathrm{M}$ et al. Factors contributing to poor results of treatment of esophageal atresia in developing countries. Pediatr Surg Int 1996;11:312-5.

17. Engum SA, Grosfeld JL, West KW et al. Analysis of morbidity and mortality in 227 cases of esophageal Indian J Pediatr 2015;82:586-90.

18. Tan Tanny SP, Fearon E, Hawley A et al. Predictors of Mortality after Primary Discharge from Hospital in Patients with Esophageal Atresia. J Pediatr 2020;219:70-5.

19. Spaggiari E, Faure G, Rousseau V et al. Performance of prenatal diagnosis in esophageal atresia. Prenat Diagn 2015;35:888-93.

20. Bradshaw CJ, Thakkar H, Knutzen L et al. Accuracy of prenatal detection of tracheoesophageal fistula and oesophageal atresia. J Pediatr Surg 2016;51:1268-72.

21. De Jong EM, De Haan MA, Gischler SJ et al. Pre- and postnatal diagnosis and outcome of fetuses and neonates with esophageal atresia and tracheoesophageal fistula. Prenat Diagn 2010;30:274-9.

22. Prasad J. Esophageal atresia and tracheoesophageal fistula with associated anomalies in a tertiary care hospital of north India. Int Surg J 2017;4:3456-60.

23. Galarreta CI, Vaida F, Bird LM. Patterns of malformation associated with esophageal atresia/tracheoesophageal fistula: A retrospective single centre study. Am J Med Genet A 2020;182:1351-63.

24. Arora M, Gupta DK. Esophageal atresia: The AIIMS experience. Textbook of Neonatal Surgery. Gupta DK, editor. Modern publishers: New Delhi 2000, 336-41.

25. Ekselius J, Salo M, Arnbjornsson E et al. Treatment and Outcome for Children with Esophageal Atresia from a Gender Perspective. Surg Res Pract 2017;2017:8345798.

26. Masuya R, Kaji T, Mukai M, Nakame K et al. Predictive factors affecting the prognosis and late complications of 73 consecutive cases of esophageal atresia at 2 centers. Pediatr Surg Int 2018;34:1027-33.

27. Mortell AE, Azizkhan RG. Esophageal atresia repair with thoracotomy: the Cincinnati contemporary experience. Semin Pediatr Surg 2009;18:12-9.

28. Spitz L. Oesophageal atresia. Orphanet J Rare Dis 2007;11(2):24.

29. Parul Naib, Kanishak Gautam, Gaurav Mishra et al. Utilization of Neo-natal Care Packages under PMJAYFindings from Preliminary Analysis [Internet]. National Health Authority. Working paper No.: 2. Available from: https://pmjay.gov.in/sites/default/files/202105/Working\%20paper\%20002$\% 20$ Utilization $\% 20$ of $\% 20 \mathrm{Neo}-$ natal\%20Care\%20Packages.pdf

30. Rattan KN, Singh J, Dalal P. Clinical profile and short-term outcome of neonates with esophageal atresia and tracheoesophageal fistula at tertiary care center in a developing country: A 25-year experience. J Clin Neonatol 2017;6:225-30.

31. Wong NA, Hunt LP, Marlow N. Risk factors for developing neonatal septicaemia at a Malaysian hospital. J Trop Pediatr 1997;43:54-8.

32. Gadgade BD, Annigeri VM, Kabbin G et al. Outcomes of 
esophageal atresia in a tier II referral centre. Int Surg J 2016;3:318-20.

33. Choudhury SR, Ashcraft KW, Sharp RJ et al. Survival of patients with esophageal atresia: influence of birth weight, cardiac anomaly, and late respiratory complications. J Pediatr Surg 1999;34:70-3. Discussion 74.

34. Askarpour Shahnam, Hafezi Mohammad-Hassan, Javaherizadeh Hazhir, Alavi Mostafa. Esophageal atresia: Our experiences in a university hospital. Annals of Pediatric Surgery 2014;10:31-4.

35. Poenaru D, Laberge JM, Neilson IR et al. A new prognostic classification for esophageal atresia. Surgery 1993;113:42632.

36. Smith C, Teng F, Branch E, Chu S, Joseph KS. Maternal and Perinatal Morbidity and Mortality Associated with Anemia in Pregnancy. Obstet Gynecol 2019;134:1234-44.

37. Rahman MM, Abe SK, Rahman MS et al. Maternal anemia and risk of adverse birth and health outcomes in low- and middle-income countries: systematic review and metaanalysis. Am J Clin Nutr 2016;103:495-504.

38. Bhardwaj N, Hasan SB, Zaheer M. Maternal care receptivity and its relation to perinatal and neonatal mortality. A rural study. Indian Pediatr 1995;32:416-23.

39. Krishna A, Murali MV, Ahuja S, Kaur N. Factors influencing survival in esophageal atresia. Indian Pediatr 1994;31:80-3. 